\title{
Review of Evidence-Based Support for Pretreatment Imaging in Melanoma
}

\author{
Michael S. Sabel, MD, and Sandra L. Wong, MD, Ann Arbor, Michigan
}

\section{Key Words}

Melanoma, radiographic imaging, chest radiograph, CT scan, PET scan, ultrasound, sentinel lymph node biopsy

\begin{abstract}
When making a new diagnosis of melanoma, clinicians often obtain imaging studies to rule out clinically occult distant disease. These studies range from inexpensive tests, such as chest radiographs, to more expensive studies, such as PET/CT. The impetus for ordering these studies is usually the desire to identify potentially resectable distant disease, avoid surgery when curative resection is not possible, and assuage patient anxiety by showing that no evidence of distant disease is present. However, some detrimental aspects to these studies are less apparent, including cost and potential for false-positive findings. Although routine use seems reasonable, the true benefit of these studies depends on the probability of clinically occult disease being present, likelihood that disease will be detected with the available technology, and impact of earlier detection on outcome. Contrary to current practice patterns, available evidence suggests that preoperative imaging studies are associated with significant costs and minimal benefit in most patients with melanoma. This article reviews available literature on the role of pretreatment imaging in patients with newly diagnosed cutaneous melanoma. (JNCCN 2009;7:281-289)
\end{abstract}

\section{Medscape: Continuing Medical Education Online}

\section{Accreditation Statement}

This activity has been planned and implemented in accordance with the Essential Areas and policies of the Accreditation Council for Continuing Medical Education through the joint sponsorship of Medscape, LLC and JNCCN - The Journal of the National Comprehensive Cancer Network. Medscape, LLC is accredited by the Accreditation Council for Continuing Medical Education (ACCME) to provide continuing medical education for physicians.

Medscape, LLC designates this educational activity for a maximum of 0.75 AMA PRA Category 1 Credits $^{\mathrm{TM}}$. Physicians should only claim credit commensurate with the extent of their participation in the activity. All other clinicians completing this activity will be issued a certificate of participation. To participate in this journal CME activity: (1) review the learning objectives and author disclosures; (2) study the education content; (3) take the post-test and/or complete the evaluation at www.medscape.com/cme/jnccn; (4) view/print certificate.

\section{Learning Objectives}

Upon completion of this activity, participants will be able to:

- Identify prognostic factors in patients with newly diagnosed melanoma

- Define the recommendations for pretreatment imaging from the National Comprehensive Cancer Network (NCCN) in patients with melanoma
From the University of Michigan Comprehensive Cancer Center, Ann Arbor, Michigan.

Submitted September 30, 2008; accepted for publication December 22, 2008.

Correspondence to: Michael S. Sabel, MD, University of Michigan Health Systems, 1500 East Medical Center Drive, 3303 Cancer Geriatric Center SPC 5932, Ann Arbor, MI 48109-5932. E-mail: msabel@med.umich.edu

\section{EDITOR}

Kerrin G. Robinson, MA, Medical/Scientific Editor, Journal of the National Comprehensive Cancer Network

Disclosure: Kerrin G. Robinson, MA, has disclosed no relevant financial relationships.

\section{AUTHORS AND CREDENTIALS}

Michael S. Sabel, MD, University of Michigan Comprehensive Cancer Center, Ann Arbor, Michigan

Disclosure: Michael S. Sabel, MD, has disclosed no relevant financial relationships.

Sandra L. Wong, MD, University of Michigan Comprehensive Cancer Center, Ann Arbor, Michigan

Disclosure: Sandra L. Wong, MD, has disclosed no relevant financial relationships.

\section{CME AUTHOR}

Désirée Lie, MD, MSEd, Clinical Professor, Family Medicine, University of California, Orange; Director, Division of Faculty Development, UCI Medical Center, Orange, California

Disclosure: Désirée Lie, MD, MSEd, has disclosed no relevant financial relationships. 
- Describe the types of pretreatment imaging techniques used in patients with melanoma

- Describe imaging techniques for identifying regional lymph node metastases in patients with newly diagnosed melanoma

- Describe biochemical tests used for assessing prognosis in patients with melanoma

Imaging studies are frequently used in workups of patients with cancer to determine extent of disease before definitive treatment recommendations are made. Various imaging modalities may allow for more accurate staging or help detect clinically occult disease. In melanoma, imaging is focused on detecting occult sites of regional or distant disease. However, recommendations for preoperative imaging must be considered in the context of sentinel lymph node biopsy (SLNB), which is a minimally invasive procedure that provides excellent staging information for patients with clinically localized disease.

Although characteristics of the primary tumor are important in determining stage and prognosis, the status of the sentinel node is the most important prognostic factor in patients with intermediate thickness melanoma. ${ }^{1}$ Experts generally agree that sentinel node biopsy should be considered in patients with clinically node-negative melanoma of $1-\mathrm{mm}$ or greater Breslow thickness, but no consensus exists as to what constitutes adequate or necessary preoperative imaging. Therefore, the decision to pursue imaging depends on several factors, including the probability of clinically occult disease being present, likelihood of disease detection given the limitations of imaging, potential for false-positive findings, and cost and morbidity of the study (including the cost of necessary follow-up examinations or biopsies).

Consensus guidelines, including those from the NCCN, reflect the relative lack of certainty about benefits from pretreatment imaging for melanoma in asymptomatic patients. ${ }^{2}$ For stage IA disease with adverse pathologic features, stage IB disease, and stage II disease, sentinel node biopsy is generally recommended. However, chest radiography (CXR) is considered optional and other imaging is not routinely recommended. The American Academy of Dermatology recommends no staging for patients with melanoma less than $4.0-\mathrm{mm}$ thick, ${ }^{3}$ whereas the British Association of Dermatologists and Melanoma Study Group for the United Kingdom recom- mend that patients with melanomas thicker than $2.0 \mathrm{~mm}$ undergo CXR, liver ultrasound, or CT of chest/abdomen and pelvis. ${ }^{4}$ This recommendation has led to an extremely wide range of practices among clinicians caring for patients with melanoma.

Part of the difficulty in assessing the role of pretreatment imaging studies is defining the reason for ordering the test. Theoretically, the end point should be obvious: whether obtaining the study identifies clinically occult disease, which could change planned management. This end point, however, is vague in many respects. Whether imaging findings would change the decision to proceed with sentinel node biopsy or completion node dissection in the case of a positive sentinel node is unclear. It is a Herculean task to determine how often a study must meet this goal to be worthwhile, especially when balanced against the host of ensuing tests that indeterminate findings would generate, which are not without cost and morbidity. Although upfront imaging in all patients could provide not only baseline information for future comparison studies but also comfort for most patients because examinations will likely be negative, it can also add significant cost and produce extreme anxiety for patients who have false-positive or indeterminate studies (Table 1).

This article reviews the role of pretreatment imaging in patients with cutaneous melanoma. Importantly, the debate over the need for and extent of imaging in patients with cutaneous melanoma is limited to those without clinical suspicion of metastatic disease. Focused imaging based on symptoms or clinical suspicion should be pursued as clinically indicated. Any patient newly diagnosed with melanoma should undergo a thorough history and physical examination, with a detailed review of systems. Although comparatively crude when considering today's available imaging technology, clinical evaluation remains one of the most important modalities in identifying distant disease. Data from studies on melanoma surveillance clearly show the importance of the history and physical in identifying recurrences.., 6

\section{Pretreatment Imaging of Patients with Clinically Node-Negative Melanoma}

\section{CXR}

CXR is the most common imaging study obtained on patients newly diagnosed with melanoma. Arguments for routine CXR include its low cost, the potential 
Pretreatment Imaging in Melanoma

need in patients undergoing general anesthesia, the benefit of having a baseline CXR if the patient has never had one before, and the fact that the lungs are among the most common visceral sites of spread. ${ }^{7,8}$

However, several studies have examined the relative worth of CXR in staging patients with melanoma, with little evidence to support its use. Khansur et al. ${ }^{9}$ found no true-positives and 2 false-positives among 72 patients with localized melanoma who underwent CXR. Ardizzoni et al. ${ }^{10}$ reported that all studies were normal among 93 patients with localized melanoma who underwent pretreatment CXR. Zartman et al. ${ }^{11}$ also found no positive results among 90 patients with asymptomatic localized disease who underwent CXR. Hofmann et al. ${ }^{5}$ reported on 661 melanoma patients, of which 524 underwent a staging CXR, finding 23 false-positives and only 1 true-positive. Cost-efficiency analysis showed that despite the low cost of CXR itself, it is a highly cost inefficient examination. Yancowitz et al. ${ }^{12}$ reported that among 131 CXRs obtained among patients with $\mathrm{T} 1 \mathrm{~b}$ to $\mathrm{T} 3 \mathrm{~b}$ disease, 7 were suspicious or highly suspicious for melanoma. Of these, none was a true-positive, whereas 5 were falsepositives ( 2 lost to follow-up). Hafner et al..$^{13}$ found 4 of 100 patients had suspicious findings on preoperative CXR, all of which were PET negative and showed no progression on follow-up imaging.

Wang et al. ${ }^{14}$ prospectively examined the CXR findings of 210 patients with no clinical evidence of regional or distant disease. The mean Breslow depth was $1.38 \mathrm{~mm}$, with it being less than $1 \mathrm{~mm}$ in $54 \%$ of patients and $1 \mathrm{~mm}$ or larger (range, $0.13-10 \mathrm{~mm}$ ) in $46 \%$ of patients. Of the 15 patients who had positive or equivocal melanoma-related results, none had confirmed lung metastases and only 1 ultimately developed metastatic disease. Thus, the false-positive rate of CXR (7\%) was considerably higher than the rate of detecting asymptomatic pulmonary disease.

Terhune et al. ${ }^{15}$ retrospectively reviewed 1032 asymptomatic patients with localized melanoma and found that $876(85 \%)$ had an initial staging CXR performed. Of these patients, $5 \%$ had melanoma in situ, $62 \%$ had a Breslow depth less than $1.5 \mathrm{~mm}$, and $25 \%$ had a thickness between 1.5 and $4.0 \mathrm{~mm} \mathrm{(3 \%}$ could not be staged). Overall, 130 (15\%) had suspicious findings that necessitated additional workup, whereas only $1(0.1 \%)$ had a true-positive finding. When the study was limited to patients with melanomas between 1.5 and $4.0 \mathrm{~mm}$, the incidence of de-

\begin{tabular}{|c|c|}
\hline \multicolumn{2}{|c|}{$\begin{array}{l}\text { Table } 1 \text { Advantages and Disadvantages of } \\
\text { Preoperative Imaging }\end{array}$} \\
\hline Advantages & Disadvantages \\
\hline Accurate staging & Cost of studies \\
\hline $\begin{array}{l}\text { Avoid unnecessary surgery } \\
\text { for those with stage IV } \\
\text { disease }\end{array}$ & $\begin{array}{l}\text { Incorrect change in surgical } \\
\text { management from false- } \\
\text { positive finding }\end{array}$ \\
\hline $\begin{array}{l}\text { Psychological benefit to } \\
\text { patient with negative } \\
\text { report }\end{array}$ & $\begin{array}{l}\text { Cost and morbidity of } \\
\text { additional studies/biopsies } \\
\text { prompted by false-positive } \\
\text { findings }\end{array}$ \\
\hline comparison & $\begin{array}{l}\text { Psychological detriment } \\
\text { to patient with an } \\
\text { indeterminate report }\end{array}$ \\
\hline
\end{tabular}

tectable pulmonary disease was $0.4 \%$.

The cost of this practice is not insignificant, particularly in the absence of evidence that finding synchronous asymptomatic pulmonary disease improves survival. When follow-up studies are considered, the conservative financial cost of CXR for the 876 patients in the Terhune et al. ${ }^{15}$ study was nearly $\$ 200,000$ to find 1 patient with metastatic disease. ${ }^{16}$ This, of course, does not consider the anxiety and stress caused to the 14 patients with false-positive findings. Other studies have found more significant cost savings associated with foregoing CXR. ${ }^{17}$ Part of the argument against routine CXR is that when melanoma metastasizes to the lungs, it usually appears as multiple small foci, often less than the threshold necessary to be detected on CXR. ${ }^{18-20}$

Many have argued that CXR should be used simply because it is widely available, relatively inexpensive, and noninvasive. ${ }^{21,22}$ However, these variables do not satisfy an argument to continue its routine used. The available evidence clearly supports no role for obtaining routine CXRs in patients with clinically node-negative melanoma with no symptoms of metastatic disease.

\section{Imaging of the Regional Lymph Node Basin}

Although SLNB is a well-established minimally invasive procedure that provides accurate staging information, several investigators have examined the use of PET scanning for detecting regional lymph nodes as an alternative to sentinel node biopsy, with sensitivity ranging from $0 \%$ to $40 \%{ }^{23-30}$ and a mean sensitivity of $16 \%$ to $17 \% .^{31,32}$ The poor sensitivity is secondary to the fact that the volume of disease within the sentinel nodes is rarely greater than the threshold necessary for detection using PET scanning. 33,34 
Hafner et al. ${ }^{13}$ examined the role of ultrasound and PET for detecting regional metastases before SLNB in 100 patients with newly diagnosed melanoma greater than $1.0 \mathrm{~mm}$. Ultimately, SLNB showed that $26 \mathrm{pa}-$ tients had regional metastases; however, only 4 had disease greater than $2 \mathrm{~mm}$. Ultrasound had a sensitivity of $8 \%$, specificity of $88 \%$, positive predictive value of $18 \%$, and negative predictive value of $73 \%$. Ultrasound had 12 false-positives and was no better than clinical examination alone. PET did not have any false-positives, with a positive predictive value of $100 \%$, but also had a sensitivity of only $8 \%$. The combination did not fare much better. Despite these negative findings, the authors concluded that, along with physical examination, ultrasound may play a role in the detecting macroscopic lymph node metastases, although this only represented about $3 \%$ of patients.

Ultrasound of the axilla before SLNB, with ultrasound-guided fine needle aspiration (FNA) of abnormal lymph nodes to confirm metastases, has been proven to be a useful approach to preoperative imaging in patients with breast cancer, and has become routine at many institutions..$^{35} \mathrm{~A}$ similar approach was examined in melanoma. Rossi et al..$^{36}$ prospectively examined preoperative regional ultrasound and FNA in 125 patients with melanoma greater than 1 mm undergoing SLNB. Ultimately, 31 patients were found to have regional metastases, with 12 detected by ultrasound-guided FNA, for a sensitivity of $39 \%$. Voit et al. ${ }^{37}$ reported a higher sensitivity (79\%) in a similar study. Based on these results, $10 \%$ and $16 \%$ of patients, respectively, would have avoided SLNB and could have proceeded directly to complete lymph node dissection.

However, although Starritt et al. ${ }^{38}$ showed that targeted ultrasound examination of sentinel lymph nodes can detect metastatic melanoma deposits as small as approximately $4.5 \mathrm{~mm}$ in diameter, ultrasound of the regional basin cannot be considered cost-effective because most metastatic melanoma deposits in sentinel lymph nodes are considerably smaller than this at initial staging. The ongoing Multicenter Selective Lymphadenectomy Trial-II (MSLT-II) will provide further data on the efficacy of regional basin ultrasound before SLNB.

Available evidence supports no role for PET scanning in identifying regional metastases in patients with clinically node-negative melanoma. The role of ultrasound of the regional basin is still under investigation.

\section{Body Imaging (CT or PET Scan)}

Evidence supporting the routine use of CT among patients with newly diagnosed and unstaged melanoma is sparse. Only 2 studies specifically examine CT scans among patients in whom the nodal stage is unknown. Yancovitz et al. ${ }^{12}$ found that among 57 of 158 patients with T1b through T3b disease who underwent CT scans, 24 suspicious areas were identified. Among these, none were true-positives and 21 were false-positives. For abdomen/pelvis CT, the results were similar: of the 11 suspicious findings identified, none were true-positives, whereas 10 were false-positives. Both suspicious findings on the 57 head CTs also turned out to be falsepositives. From this same dataset, 42 patients underwent PET/CT scans. Of 5 suspicious lesions identified, 1 was a true-positive, whereas 3 were false-positives.

Buzaid et al. ${ }^{39}$ retrospectively looked at the use of CT scans among 151 asymptomatic patients with stage I, II, or III melanoma. Among the 29 patients who had suspicious findings, 24 were benign, 2 truly represented distant disease, and 3 had second primary tumors identified. The authors concluded that preoperative CT scans had minimal benefit, and indicated that additional and sometimes invasive studies were often needed that had the potential to increase patient anxiety.

Therefore, experts have suggested that more sensitive screening tests, despite their increased cost, may have a greater impact on screening patients with asymptomatic melanoma. In addition to its use in detecting regional disease, fluorodeoxyglucose (FDG)PET has been examined as a method of identifying distant disease in patients with clinically node-negative disease. Several studies have touted the role of PET scanning in identifying distant disease; however, many of these positive findings include the detection of clinically palpable regional disease, and many studies include patients with stage IV melanoma. When limited to patients with asymptomatic, clinically node-negative melanoma, the use of FDG-PET is not strongly supported. Among 609 patients staged using FDG-PET (pooled from several studies), 38 patients (6\%) had abnormal uptake outside the primary site or regional nodes, but only 1 had a true melanoma metastasis. ${ }^{32}$ Most patients had false-positive findings, although unrelated neoplastic processes were identified in 10 . In addition to being inferior to SLNB in detecting regional metastases, FDG-PET does not identify distant disease in asymptomatic patients. 
The available evidence clearly supports no role for obtaining either routine CT or PET scan in patients with clinically node-negative melanoma without symptoms of metastatic disease. The staging information obtained from intraoperative lymphatic mapping and sentinel node biopsy is likely to yield more accurate and important prognostic information. ${ }^{40}$

An alternative strategy to routine testing is selective testing using serum markers to identify patients with a higher risk for distant disease. One of the most common staging tests obtained in patients with melanoma is the serum lactate dehydrogenase (LDH) level. However, the efficacy of an elevated serum $\mathrm{LDH}$ in selecting patients who do not harbor metastases seems limited, because LDH only has prognostic value in stage IV disease. Wang et al. ${ }^{14}$ prospectively examined the use of serum $\mathrm{LDH}$ in 96 patients with melanoma $1 \mathrm{~mm}$ or greater. They found that elevations were not uncommon (15\%) but did not lead to the detection of systemic disease.

Strobel et al. ${ }^{41}$ looked at limiting PET/CT scan only to patients with an elevated serum S-100B. This tumor marker may reflect the tumor burden and be useful for monitoring therapy in stage IV disease. Screening 165 patients with high-risk melanoma (defined as Breslow thickness $>4.0 \mathrm{~mm}$, Clark level III or IV, or known resected metastases) yielded elevated serum S-100B levels in 47 patients. In this case, PET/CT performed well, correctly identifying metastases in 38 of these high-risk patients. However, S-100B did have a high number of false-positive results $(17 \%)$. The authors recommend that, if S-100B is used, it should be repeated 2 to 4 weeks after the initial determination if no clinical evidence of distant disease is present. Additional studies are needed to confirm the efficacy of serum S-100B levels in the routine staging of patients with melanoma.

\section{Imaging After SLNB}

For patients with clinically node-negative melanoma, minimal evidence supports obtaining CXR, CT, or PET before proceeding with wide excision and, if indicated, SLNB. Patients with a positive sentinel lymph node will typically return to the operating room for a completion lymph node dissection, and therefore the question arises whether obtaining imaging at this juncture might be worthwhile. $\mathrm{Pa}$ tients with known nodal metastasis have a significantly higher risk for distant disease and represent a select group of patients with different treatment decisions. ${ }^{40}$ Several authors have examined the paradigm of proceeding with SLNB and limiting imaging to patients with metastatic disease identified in the sentinel lymph node. Theoretically, discovery of occult distant metastases may preclude consideration of completion lymphadenectomy. ${ }^{42}$ However, studies have found that this imaging rarely changes plans for completion lymphadenectomy, and may actually delay surgical treatment of regional disease.

Constantinidou et al..$^{43}$ performed either PET scan or PET/CT in 30 patients with a positive sentinel lymph node and a melanoma of Breslow depth greater than $1 \mathrm{~mm}$. Only 2 patients had positive findings and neither was melanoma-related. In a retrospective analysis of complete radiologic staging (CXR; CT of the chest, abdomen, and pelvis; and CT or MRI of the brain) in patients with positive sentinel lymph nodes, Miranda et al..$^{42}$ found disease in $0.5 \%$.

In an examination of CT and/or MRI in 270 asymptomatic patients with a positive sentinel lymph node, Aloia et al. ${ }^{44}$ found disease in only $1.9 \%$. Although this analysis showed fewer false-positive results than previous studies, which the authors attributed to improved technology, these were still frequent (12\%). The authors proposed that further selection of patients for imaging based on the characteristics of the primary tumor and sentinel lymph node (T4, ulceration, large sentinel lymph node tumor burden) may be reasonable, as much to obtain baseline data in patients at high risk for recurrence as to find occult metastases.

Gold et al. ${ }^{45}$ retrospectively examined various radiologic studies obtained after a positive SLNB, and also found that indeterminate findings are high but distant disease is rarely found $(3.7 \%)$. The impact, however, is more significant in patients with thick melanomas and macrometastases in the sentinel lymph node. Had CT or PET been limited only to patients with both thick melanomas and macrometastases in the sentinel lymph node, the frequency of positive studies would have been $16 \%{ }^{45}$

These retrospective studies have shown a lack of consistency in the types of imaging obtained. Common sites of metastases include the brain, lung, gastrointestinal tract, and distant nodal basins. Therefore, imaging studies are often used to examine the head, thorax, abdomen, and pelvis. In these studies, significant variation is seen in the imaging studies obtained. For example, brain imaging was variously performed with CT or MRI. CT scans included chest only, abdomen/ 
pelvis only, or chest/abdomen/pelvis. CT scans of the neck were also occasionally obtained. However, head CT in asymptomatic patients, chest CT in patients with lower-extremity melanomas/inguinal metastases, and pelvic CT in patients with axillary or cervical adenopathy are not indicated. ${ }^{46}$ Functional imaging, such as 18FDG-PET scans, are frequently added to crosssectional imaging studies. PET scans were used as a sole imaging modality or in conjunction with standard CT scans. However, with the advent of fusion PET-CT scans, which provide corresponding noncontrasted CT images, these are sometimes used instead of standard CT scans of the chest, abdomen, and pelvis.

Available evidence suggests that although routine CT or PET for patients with positive sentinel lymph nodes does not seem worthwhile, it may have a role in patients with thick primary tumors and a large tumor burden in the sentinel lymph node. Further prospective studies are needed to delineate the ideal subset of patients and cost-effectiveness of this approach.

\section{Pretreatment Imaging of Patients With Clinically Node-Positive Melanoma or Resectable Stage IV Disease}

In an initial retrospective review of CT scans among patients with stage I, II, and III melanoma, ${ }^{39}$ preoperative CT scans identified synchronous distant disease in 1 of 23 who presented with clinically evident regional metastases. In a second study, the authors looked specifically at the CT results of 89 asymptomatic patients with clinical locoregional disease and normal CXR and serum LDH levels. ${ }^{47} \mathrm{CT}$ scanning identified metastatic disease in 6 patients $(7 \%)$, whereas 20 (22\%) had false-positive findings. Although the false-positive rate is concerning and biopsies should be used to confirm suspicious findings, CT was useful, particularly in patients with melanoma below the waist or in the head and neck region.

Kuvshinoff et al. ${ }^{46}$ reported similar findings when examining the role of CT in 347 patients with clinical stage III disease, finding detection and false-positive rates of $4.2 \%$ and $8.4 \%$, respectively. Chest CT was particularly useful in patients with cervical adenopathy, whereas pelvic CT was most helpful in those with groin adenopathy. Minimal benefit was seen in patients with axillary adenopathy. The authors therefore recommended a selective approach to CT scans in patients with palpable adenopathy. Johnson et al. ${ }^{48}$ examined CT use among patients with stage III disease, most of whom (99) had clinically node-positive disease, whereas elective node dissection identified 28 as having stage III disease. Although they found the yield of CT to be low, it was not insignificant and could alter treatment decisions.

Several authors have examined the use of PET scanning in staging patients with more advanced melanoma being considered for surgical resection. Tyler et al. ${ }^{49}$ examined 106 PET scans among 95 patients with clinically palpable nodal disease. Findings on PET scans led to a change in the planned clinical management in $15 \%$ of cases. Damian et al. ${ }^{50}$ reported a $22 \%$ alteration in patient management with PET scan. Brady et al. ${ }^{51}$ examined the combination of PET and CT in the preoperative setting for patients with more advanced disease (AJCC stage IIC, III, or IV) and found that not only was the combination more effective than either modality alone, but the use of PET-CT led to a change in clinical management in $35 \%$ of patients. Other studies have also shown PET-CT to be superior to PET alone. ${ }^{52,53}$ In a retrospective analysis of 250 consecutive patients, the combination of PET-CT detected more metastases than either PET or CT alone..$^{53}$ Strobel et al. ${ }^{54}$ prospectively examined PET scanning with and without dedicated CT readout and found improved sensitivity, specificity, and accuracy with dedicated CT interpretation. They concluded that a dedicated analysis of CT data significantly improves the results.

All of these studies, however, found that falsepositive findings were still significant. In several cases, changes in management were made incorrectly because of false-positive findings. ${ }^{51,55}$ Therefore, histologic confirmation of suspected metastases must be performed before the treatment plan is altered.

\section{Conclusions}

When a patient is newly diagnosed with melanoma, often the patient and physician have a strong desire to order preoperative staging studies to rule out distant disease before proceeding with curative surgery. However, the negative side to this approach is not immediately apparent, nor insignificant, and the evidence supports a much more selective use of imaging studies. Figure 1 presents a reasonable algorithm for radiographic staging of patients with melanoma based on the available evidence.

The single most important staging study in pa- 


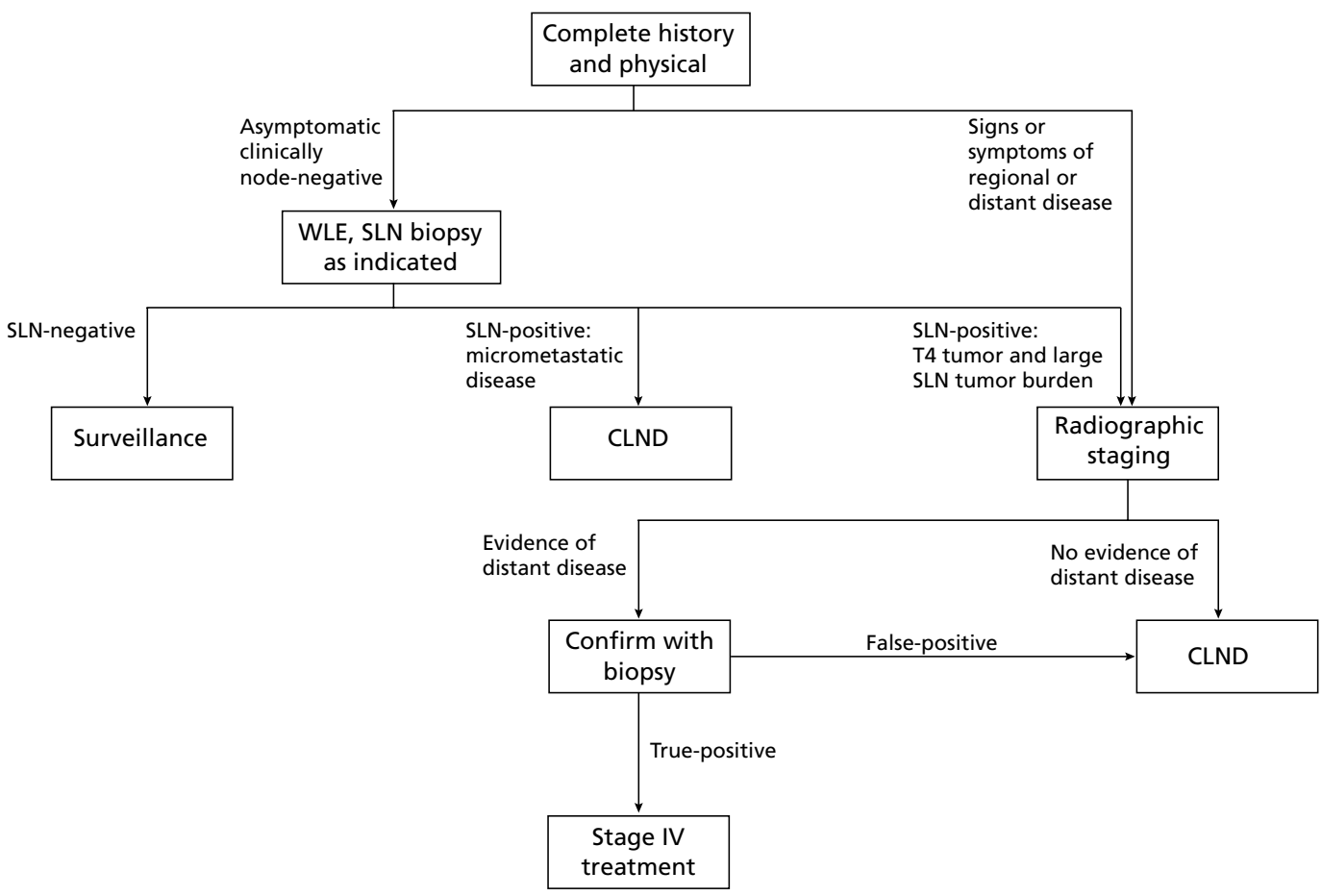

Figure 1 Proposed algorithm for preoperative imaging of patients with cutaneous melanoma based on the current evidence. Abbreviations: CLND, completion lymph node dissection; SLN, sentinel lymph node; WLE, wide local excision.

tients with newly diagnosed melanoma is a thorough history and physical examination. Any patient with signs or symptoms should undergo radiographic staging. Patients with evidence of nodal or in-transit metastases seem to benefit from radiographic staging before proceeding with resection, both in avoiding surgery in futile cases and planning the extent of the operation. Asymptomatic patients with clinically negative nodes derive minimal if any benefit from CXR, CT, PET, or regional ultrasound, and should proceed with wide local excision and SLNB.

Most patients with a positive sentinel lymph node should still proceed with completion node dissection without imaging; however, staging patients at a higher risk for distant disease may be reasonable, specifically those with thick primary tumors and a large tumor burden in the sentinel lymph node. It is important to remember the high false-positive rate associated with all of the available imaging studies and confirm any suspected areas of distant disease using biopsy before abandoning a potentially curative operation. Further prospective studies of preoperative imaging are necessary to identify the optimal subset of asymptomatic patients who should undergo preoperative imaging and define the most appropriate studies.

\section{References}

1. Balch CM, Soong SJ, Gershenwald JE, et al. Prognostic factors analysis of 17,600 melanoma patients: validation of the American Joint Committee on Cancer melanoma staging system. J Clin Oncol 2001;19:3622-3634.

2. Coit DG, Andtbacka R, Bichakjian CK, et al. NCCN Clinical Practice Guidelines in Oncology: Melanoma, version 2, 2009. Available at: http://www.nccn.org/professionals/physician_gls/f_ guidelines.asp. Accessed January 12, 2009.

3. Sober AJ, Chuang TY, Duvic M, et al. Guideline of care for primary cutaneous melanoma. J Am Acad Dermatol 2001;45:579-586.

4. Roberts DLL, Anstey AV, Barlow RJ, et al. U.K. guidelines for the management of cutaneous melanoma. Br J Dermatol 2003;146:7-17.

5. Hofmann U, Szedlak M, Rittgen W, et al. Primary staging and follow-up in melanoma patients: monocenter evaluation of methods, costs and patient survival. Br J Cancer 2002;87:151-157.

6. Weiss M, Loprinzi CL, Creagan ET, et al. Utility of follow-up tests for detecting recurrent disease in patients with malignant melanomas. JAMA 1995;274:1703-1705.

7. Leiter U, Meier F, Schittek B, Garbe C. The natural course of cutaneous melanoma. J Surg Oncol 2004;86:172-178.

8. Patel JK, Didolkar MS, Pickren JW, Moore RH. Metastatic pattern of malignant melanoma. A study of 216 autopsy cases. Am J Surg 1978;135:807-810.

9. Khansur T, Sanders J, Das SK. Evaluation of staging workup in malignant melanoma. Arch Surg 1989;124:847-849.

10. Ardizzoni A, Grimaldi A, Repetto L, et al. Stage I-II melanoma: the value of metastatic work-up. Oncology 1987;44:87-89.

11. Zartman GM, Thomas MR, Robinson WA. Metastatic disease in patients with newly diagnosed malignant melanoma. J Surg Oncol 1987;35:163-164.

12. Yancowitz M, Finelt N, Warycha MA, et al. Role of radiologic 
imaging at the time of initial diagnosis of stage T1b-T3b melanoma. Cancer 2007;110:1107-1113.

13. Hafner J, Schmid $M H$, Kempf W, et al. Baseline staging in cutaneous malignant melanoma. Br J Dermatol 2004;150:677-686.

14. Wang TS, Johnson TM, Cascade PN, et al. Evaluation of staging chest radiographs and serum lactate dehydrogenase for localized melanoma. J Am Acad Dermatol 2004;51:399-405.

15. Terhune MH, Swanson N, Johnson TM. Use of chest radiography in the initial evaluation of patients with localized melanoma. Arch Dermatol 1998;134:569-572.

16. Kanzler MH. Initial evaluation of melanoma (letter to the editor). Arch Dermatol 1999;135:1121-1122.

17. Weiss M, Loprinzi CL, Creagan ET, et al. Utility of follow-up tests for detecting recurrent disease in patients with malignant melanoma. JAMA 1995;274:1703-1705.

18. Webb WR, Gamsu G. Thoracic metastasis in malignant melanoma: a radiographic survey of 66 patients. Chest 1977;71:176-181.

19. Chen JT, Dahmash NS, Ravin CE, et al. Metastatic melanoma to the thorax. AJR Am J Roentgenol 1981;137:293-298.

20. Das Gupta T, Brasfield R. Metastatic melanoma. Cancer 1964; 17:1323-1339.

21. Gross EA. Initial evaluation of melanoma: Don't stop getting that chest x-ray....yet. Arch Dermatol 1998; 134:623-624.

22. Parish LC, Witkowski JA. Melanoma and the radiograph: sanctity of human life. Lancet 1998; 352:922-923.

23. Klein M, Freedman N, Lotem M, et al. Contribution of whole body F-18-FDG-PET and lymphoscintigraphy to the assessment of regional and distant metastases in cutaneous malignant melanoma. Nuklearmedizin 2000;39:56-61.

24. Belhocine T, Pierard G, De Labrassinne M, et al. Staging of regional nodes in AJCC stage I and II melanoma: 18FDG PET imaging versus sentinel node detection. Oncologist 2002;7:271-278.

25. Fink AM, Holle-Robatsch S, Herzog N, et al. Positron emission tomography is not useful in detecting metastasis in the sentinel lymph node in patients with primary malignant melanoma stage I and II. Melanoma Res 2004;14:141-145.

26. Longo MI, Lazaro P, Bueno C, et al. Flourodeoxyglucose-positron emission tomography imaging versus sentinel node biopsy in the primary staging of melanoma patients. Dermatol Surg 2003; 29:245-248.

27. Libberecht $K$, Husada $G$, Peeters $T$, et al. Initial staging of malignant melanoma by positron emission tomography and sentinel node biopsy. Acta Chir Belg 2005;105:621-625.

28. Vereecken $P$, Laporte $M$, Petein $M$, et al. Evaluation of extensive intitial staging procedure in intermediate/high-risk melanoma patients. J Eur Acad Dermatol Venereol 2005;19:66-73.

29. Clark PB, Soo V, Kraas J, et al. Futility of flourodeoxyglucose F18 positron emission tomography in initial evaluation of patients with T2 to T4 melanoma. Arch Surg 2006;141:284-288.

30. Kell MR, Ridge JA, Joseph N, Sigurdson ER. PET CT imaging in patients undergoing sentinel node biopsy for melanoma. Eur J Surg Onc 2007;33:911-913.

31. Essner R, Belhocine T, Scott AM, Even-Sapir E. Novel imaging techniques in melanoma. Surg Oncol Clin N Am 2006;15:253-283.

32. Ho Shon IA, Chung DK, Saw RP, Thompason JF. Imaging in cutaneous melanoma. Nucl Med Commun 2008;29:847-876.

33. Crippa F, Leutner M, Belli F, et al. Which kinds of lymph node metastases can FDG PET detect? A clinical study in melanoma. J Nucl Med 2000;41:1491-1494.

34. Wagner JD, Schauwecker D, Davidson D, et al. Inefficacy of F-18 flourodeoxy-D-glucose-positron emission tomography scans for initial evaluation in early-stage cutaneous melanoma. Cancer 2005;104:570-579.

35. Alvarez S, Anorbe E, Alcorta P, et al. Role of sonography in the diagnosis of axillary lymph node metastases in breast cancer: a systematic review. AJR Am J Roentgenol 2006;186:1342-1348.
36. Rossi CR, Mocellin S, Scagnet B, et al. The role of preoperative ultrasound scan in detecting lymph node metastasis before sentinel node biopsy in melanoma patients. J Surg Oncol 2003;82:80-84.

37. Voit C, Kron M, Schafer G, et al. Ultrasound-guided fine needle aspiration cytology prior to sentinel lymph node biopsy in melanoma patients. Ann Surg Oncol 2006;13:1682-1689.

38. Starritt EC, Uren RF, Scolyer RA, et al. Ultrasound examination of sentinel nodes in the initial assessment of patients with primary cutaneous melanoma. Ann Surg Oncol 2005;12:18-23.

39. Buzaid AC, Sandler AB, Mani S, et al. Role of computed tomography in the staging of primary melanoma. J Clin Oncol 1993;11:638-643.

40. Morton DL, Thompson JF, Cochran AJ, et al. Sentinel-node biopsy or nodal observation in melanoma. N Engl J Med 2006; 355:1307-1317.

41. Strobel K, Skalsky J, Kalff V, et al. Tumour assessment in advanced melanoma: value of FDG-PET/CT in patients with elevated serum S-100B. Eur J Nucl Med Mol Imaging 2007;34:1366-1375.

42. Miranda EP, Gertner M, Wall J, et al. Routine imaging of asymptomatic melanoma patients with metastasis to sentinel lymph nodes rarely identifies systemic disease. Arch Surg 2004;139:831-837.

43. Constantinidou A, Hofman M, O'Doherty M, et al. Routine positive emission tomography and positron emission tomography/ computed tomography in melanoma staging with positive sentinel node biopsy is of limited benefit. Melanoma Res 2008;18:56-60.

44. Aloia TA, Gershenwald JE, Andtbacka RH, et al. Utility of computed tomography and magnetic resonance staging in patients with stage III melanoma diagnosed by sentinel lymphadenectomy. J Clin Oncol 2006;24:2858-2865.

45. Gold JS, Jaques DP, Busam KJ, et al. Yield and predictors of radiologic studies for identifying distant metastases in melanoma patients with a positive sentinel lymph node biopsy. Ann Surg Oncol 2007;14:2133-2140.

46. Kuvshinoff BW, Kurtz C, Coit DG. Computed tomography in evaluation of patients with stage III melanoma. Ann Surg Oncol 1997;4:252-258.

47. Buzaid AC, Tinoco L, Ross MI, et al. Role of computed tomography in the staging of patients with local-regional metastases of melanoma. J Clin Oncol 1995;13:2104-2108.

48. Johnson TM, Fader DJ, Chang AE, et al. Computed tomography in staging of patients with melanoma metastatic to the regional nodes. Ann Surg Oncol 1997;4:396-402.

49. Tyler DS, Onaitis M, Kherani A, et al. Positron emission tomography scanning in malignant melanoma. Clinical utility in patients with stage III disease. Cancer 2000;89:1019-1025.

50. Damian DL, Fulham MJ, Thompson E, Thompson JF. Positron emission tomography in the detection and management of metatatic melanoma. Melanoma Res 1996;6:325-329.

51. Brady MS, Akhurst T, Spanknebel K, et al. Utility of preoperative [(18)F flourodeoxyglucose-positron emission tomography scanning in high-risk melanoma patients. Ann Surg Oncol 2006;13:525-532.

52. Pfannenberg C, Aschoff P, Schanz S, et al. Propspective comparison of (18)F flouroudeoxyglucose positron emission tomography/ computed tomography and whole-body magnetic resonance imaging in staging of advanced malignant melanoma. Eur J Cancer 2007;43:557-564.

53. Reinhardt MJ, Joe AY, Jaeger U, et al. Diagnostic performance of whole body dual modality 18F-FDG PET/CT imaging for $\mathrm{N}$ - and M-staging of malignant melanoma: experience with 250 consecutive patients. J Clin Oncol 2006;24:1178-1187.

54. Strobel K, Dummer R, Husarik DB, et al. High risk melanoma: accuracy of FDG PET/CT with added CT morphologic information for detection of metastases. Radiology 2007;244:566-574.

55. Harris MT, Berlangieri SU, Cebon JS, et al. Impact of 2-deoxy2[F-18] fluoro-D-glucose postiron emission tomography on the management of patients with advanced melanoma. Mol Imaging Biol 2005; 7:304-308. 


\section{Pretreatment Imaging in Melanoma}

To obtain credit, you should first read the journal article. After reading the article, you should be able to answer the following, related, multiple-choice questions. To complete the questions and earn continuing medical education (CME) credit, please go to http://www.medscape. com/cme/jnccn.

Credit cannot be obtained for tests completed on paper, although you may use the worksheet below to keep a record of your answers. You must be a registered user on Medscape.com. If you are not registered on Medscape. com, please click on the New Users: Free Registration link on the left hand side of the website to register.

Only one answer is correct for each question. Once you successfully answer all post-test questions you will be able to view and/or print your certificate. For questions re- garding the content of this activity, contact the accredited provider, CME@medscape.net. For technical assistance, contact CME@webmd.net.

American Medical Association's Physician's Recognition Award (AMA PRA) credits are accepted in the US as evidence of participation in CME activities. For further information on this award, please refer to http://www.ama-assn. org/ama/pub/category/2922.html. The AMA has determined that physicians not licensed in the US who participate in this CME activity are eligible for AMA PRA Category 1 Cred$i t s^{\mathrm{TM}}$. Through agreements that the AMA has made with agencies in some countries, AMA PRA credit is acceptable as evidence of participation in CME activities. If you are not licensed in the U.S. and want to obtain an AMA PRA CME credit, please complete the questions online, print the certifcate and present it to your national medical association.
1. Which of the following is the most important prognostic factor in patients with newly diagnosed intermediate-risk melanoma?
A. Family history
B. Palpable regional lymph nodes
C. Sentinel node biopsy findings
D. None of the above

2. Which of the following most closely reflects the recommendations of the National Comprehensive Cancer Network (NCCN) for pretreatment assessment in asymptomatic patients with stages IA, IB, and II melanoma at diagnosis?
A. Positron emission tomography/computed tomography (PET/CT)
B. Sentinel lymph node biopsy
C. Liver ultrasound
D. All of the above

3. Which of the following is the most commonly ordered imaging study in patients with newly diagnosed cutaneous melanoma?
A. Liver ultrasound
B. Bone scan
C. Chest x-ray
D. PET scan of the lymph nodes

4. Which of the following imaging studies is highly recommended for identifying regional metastases in patients with clinically node-negative melanoma?
A. Targeted ultrasound
B. PET scan
C. Magnetic resonance imaging (MRI)
D. None of the above

5. In patients with melanoma, the serum lactate dehydrogenase ( $\mathrm{LDH}$ ) level has been found to be of prognostic value in which of the following stages of disease?
A. Stage II
B. Stage III
C. Stage IV
D. All of the above

\section{Activity Evaluation}

1. The activity supported the learning objectives. $\begin{array}{lllr}\text { Strongly Disagree } & & \text { Strongly Agree } \\ 1 & 2 & 3 & 4\end{array}$

2. The material was organized clearly for learning to occur.
Strongly Disagree
1 2 3

3. The content learned from this activity will impact my practice. $\begin{array}{lllr}\text { Strongly Disagree } & & \text { Strongly Agree } \\ 1 & 2 & 3 & 4\end{array}$

4. The activity was presented objectively and free of commercial bias. Strongly Disagree Strongly Agree $\begin{array}{lllll}1 & 2 & 3 & 4 & 5\end{array}$

To obtain credit, visit Medscape online at www.medscape.com/cme/jnccn. 